\title{
On the peculiarities of anti-plane surface waves propagation for media with microstructured coating
}

\author{
Victor A. Eremeyev ${ }^{1,2, *}$ \\ ${ }^{1}$ Gdańsk University of Technology, Faculty of Civil and Environmental Engineering, Gdańsk, \\ 80-233 Poland \\ ${ }^{2}$ Southern Federal University, Institute of Mathematics, Mechanics and Computer Science, \\ Rostov-on-Don, 344090 Russia
}

\begin{abstract}
We discuss new type of surface waves which exist in elastic media with surface energy. Here we present the model of a coating made of polymeric brush. From the physical point of view the considered model of surface elasticity describes a highly anisotropic surface coating. Here the surface energy model could be treated as $2 \mathrm{D}$ reduced strain gradient continuum as surface strain energy depends on few second spatial derivatives of displacements. From the mechanical point of view the proposed model relates to $2 \mathrm{D}$ coating made of long fibers undergoing stretching and bending deformations. We consider here anti-plane surface waves. The dispersion relation is derived and its dependence on the material parameters is analysed.
\end{abstract}

\section{Introduction}

Surface waves play an important role in mechanics of solids and fluids. In particular, such waves may be used for manufacturing of various devices of acoustoelectronics as they may carry information on the material properties and their changes in the vicinity of the free surface [1-3]. Recently, with the development of the nanotechnologies it is well-established that the material behaviour at the nanoscale may be significantly different in comparison with the same material behaviour in the bulk. In particular, positive or negative size-effect can be observed at the nanoscale. Among various approaches used for material modelling at the nanoscale the theory of surface elasticity is widely applied, see, e.g., [4-6]. In the literature are known various approaches to description of surface effects, see, e.g., GurtinMurdoch model [7, 8], Steigmann-Ogden model [9, 10], and others, see [6]. The characteristic feature of these models is the presence of surface stresses which generalize the surface tension for solids. Surface stresses may significantly change the behaviour of solutions of corresponding boundary-value problems. In particular, unlike classic linear isotropic elasticity [1] there exists new class of surface anti-plane waves that are shear waves decaying exponentially with the depth $[11,12]$.

\footnotetext{
* Corresponding author: victor.eremeev@pg.edu.pl, eremeyev.victor@gmail.com
} 
Here we discuss new type of surface anti-plane waves localized near the surface an elastic half-space considering surface strain and kinetic energies. Motivating by discrete model of polymeric brushes we proposed reduced surface elasticity model. The elastic energy of the brush is described with the use of the Stockmayer potential [13-15]. The latter is a Lennard-Jones potential with additional term responsible for dipole interactions between neighbouring elements of polymeric chains. As a result, we get in the model translational and rotational interactions between chains. Using the homogenization approach discussed in [16-18] for fiber-reinforced materials, we obtain the elastic parameters used in the surface elasticity.

The paper is organized as follows. First we recall the solution form for anti-plane deformations of a half-space. Then we introduce new model of surface elasticity and derive the corresponding boundary conditions with use of the least action principle. Finally, the dispersion relations are derived and their dependence on the material parameters is analysed.

\section{Anti-plane deformations of an elastic half-space}

\subsection{Anti-plane solutions in the bulk}

Let us consider an elastic half-space $x_{3} \leq 0$. Hereinafter $x_{k}, k=1,2,3$, are Cartesian coordinates whereas $\mathbf{i}_{k}$ are the corresponding base vectors. The anti-plane deformations of the half-space take one of the forms [1]

$$
\mathbf{u}=u_{1}\left(x_{2}, x_{3}, t\right) \mathbf{i}_{1}, \quad \text { or } \quad \mathbf{u}=u_{2}\left(x_{1}, x_{3}, t\right) \mathbf{i}_{2},
$$

where $\mathbf{u}$ is the displacements vector and $t$ is time.

For anti-plane deformations the motion equations reduce into wave equations with respect to $u_{1}$ and $u_{2}$

$$
\begin{aligned}
& \mu\left(\partial_{2}^{2}+\partial_{3}^{2}\right) u_{1}=\varrho \partial_{\mathrm{t}}^{2} u_{1}, \\
& \mu\left(\partial_{1}^{2}+\partial_{3}^{2}\right) u_{2}=\varrho \partial_{\mathrm{t}}^{2} u_{2} .
\end{aligned}
$$

For brevity here we use the following notations: $\partial_{k}$ denotes the partial derivative with respect to $x_{k}, \partial_{\mathrm{t}}$ stands for the derivative with respect to $t, \mu$ and $\varrho$ are the shear modulus and mass density, respectively.

Assuming steady-state behaviour we are looking for solution of (2) and (3) in the form

$$
u_{\alpha}=U_{\alpha} \exp (i \omega t)
$$

where $\omega$ is a circular frequency, $i$ is imaginary unit, and $U_{\alpha}$ is amplitude. As a result, (2) and (3) take the form

$$
\begin{aligned}
& \mu\left(\partial_{2}{ }^{2}+\partial_{3}{ }^{2}\right) U_{1}=-\varrho \omega^{2} U_{1}, \\
& \mu\left(\partial_{1}{ }^{2}+\partial_{3}{ }^{2}\right) U_{2}=-\varrho \omega^{2} U_{2} .
\end{aligned}
$$

Decaying with the half-space depth solutions of (5) and (6) are given by

$$
U_{1}=U_{01} \exp \left(\lambda x_{3}\right) \exp \left(i k x_{2}\right), \quad U_{2}=U_{02} \exp \left(\lambda x_{3}\right) \exp \left(i k x_{1}\right),
$$

where $\lambda=\lambda(\mathrm{k}, \omega) \equiv\left(k^{2}-\mathrm{Q} / \mu \omega^{2}\right)^{1 / 2}, \mathrm{k}$ is a wavenumber, $U_{0 \alpha}$ are constants, $\alpha=1,2$. 
Nontrivial solution in form (7) exists if and only if it satisfies to the corresponding boundary conditions at $x_{3}=0$. The latter lead to a dispersion relation that is dependence between $k$ and $\omega$. It is known that for the classic linear elasticity with free surface that relates to the boundary condition

$$
\mu \partial_{3} u_{\alpha}=0
$$

anti-plane surface waves do not exist [1].

\subsection{Surface elasticity model}

Here we consider an elastic coating made of ordered polymeric chains attached along the surface. Chains interact each other and with the substrate according to the Stockmayer potential. For the theory of polymeric brushes we refer to [13-15] and the reference therein. From the mechanical point of view such coating can be represented as a discrete system of parallel long fibers. Each fiber can be modelled as an elastic beam. For simplicity let us assume that the fibers are oriented along $x_{1}$-axis. After averaging procedure as discussed in [16-18] we obtain the surface strain energy density. Under infinitesimal anti-plane deformations it has the form

$$
2 W=K_{s}\left[\left(\partial_{2} u_{1}\right)^{2}+\left(\partial_{1} u_{2}\right)^{2}\right]+K_{b}\left(\partial_{1}^{2} u_{2}\right)^{2},
$$

where $K_{s}$ and $K_{b}$ are positive elastic surface moduli related to the shear and bending deformations, respectively. Obviously, (9) presents highly anisotropic media as the energy depends on the spatial derivatives of displacements of different order

In addition we introduce the surface kinetic energy as in the Gurtin-Murdoch model [8]

$$
2 K=m\left[\left(\partial_{\mathrm{t}} u_{1}\right)^{2}+\left(\partial_{\mathrm{t}} u_{2}\right)^{2}\right]
$$

where $m$ is the surface mass density.

\subsection{Boundary conditions}

In order to get the proper boundary conditions we apply the variational technique based on the stationarity of the least action functional, see, e.g., [11, 12] for details. With (9) and (10) we get the following boundary conditions

$$
\begin{gathered}
\mu \partial_{3} u_{1}=-m \partial_{\mathrm{t}}^{2} u_{1}+K_{s} \partial_{2}{ }^{2} u_{1}, \\
\mu \partial_{3} u_{2}=-m \partial_{\mathrm{t}}^{2} u_{2}+K_{s} \partial_{1}{ }^{2} u_{2}-K_{b} \partial_{1}{ }^{4} u_{2},
\end{gathered}
$$

which are valid for (2) and (3), respectively. Eq. (11) corresponds to the Gurtin-Murdoch model for anti-plane deformations [11], whereas (12) includes additional term describing the bending energy as in the Steigmann-Ogden model [10]. The model (9) and (10) results in different boundary conditions at the free surface depending on the direction of the surface wave propagation. Let us note that (12) is a special case of the weakly nonlocal models of surface elasticity introduced in [19] within the framework of the direct approach.

\subsection{Dispersion relations}

Substituting (7) into (11) and (12) we have the dispersion relations

$$
\mu \lambda(k, \omega)=m \omega^{2}-K_{s} k^{2}
$$




$$
\mu \lambda(k, \omega)=\mathrm{m} \omega^{2}-K_{s} k^{2}-K_{b} k^{4} .
$$

Dispersion relation (13) was analysed in details in [11], whereas (14) was analysed in [19]. Let us note that the presence of the term $K_{b} k^{4}$ changes the behaviour of dispersion curves, see [19] for details.

\section{Conclusions}

Here we present new model of surface elasticity under small deformations. Unlike GurtinMurdoch [7, 8] and Steigmann-Ogden [9, 10] models of surface elasticity the presented strain energy density corresponds to new strongly anisotropic model of surface elasticity, which can be treated as one-dimensional extension of the Gurtin-Murdoch model towards Steigmann-Ogden model of surface elasticity since the strain energy has term related with the bending energy, but only in one direction. The presented elastic model is an example of two-dimensional anisotropic strain-gradient media with different differential properties in different directions: it possesses bending stiffness only in one direction. The model can be interpreted as an elastic membrane reinforced by a family of ordered elastic beams. There are some similarities with recently proposed metamaterials based on pantographic beam lattices, see [16-18, 20] and review [21].

Considering anti-plane deformations within the proposed model we discuss here the propagation of surface anti-plane waves that is also known as shear horizontal (SH-) waves, which are polarized perpendicularly with respect to the sagittal planes. The dispersion relations are derived. It is interesting that the dispersion properties depend on the direction of the wave propagation.

The Author acknowledges financial support from the Russian Science Foundation under the grant No 15-19-10008-P.

\section{References}

1. J. Achenbach, Wave propagation in elastic solids (North Holland, Amsterdam, 1973)

2. W. M. Ewing, W. S. Jardetzky, F. Press, Elastic waves in layered media (McGrawHill, New York, 1957)

3. H. Überall, Physical acoustics, W. P. Mason, R. N. Thurston (Eds.), Vol. X, (Academic Press, New York, 1973)

4. H. L. Duan, J. Wang, B. L. Karihaloo, Adv. Appl. Mech., 42, Elsevier, 1 (2008)

5. J. Wang, Z. Huang, H. Duan, S. Yu, X. Feng, G. Wang, W. Zhang, T. Wang, Acta Mech. Solida Sin., 24, 52 (2011)

6. V. A. Eremeyev, Acta Mech., 227, 29 (2016)

7. M. E. Gurtin, A. I. Murdoch, Arch. Ration. Mech. An., 57, 291 (1975)

8. M. E. Gurtin, A. I. Murdoch, Int. J. Sol. Struct., 14, 431 (1978)

9. D. J. Steigmann, R. W. Ogden, Proc. Roy. Soc. A, 453, 853 (1997)

10. D. J. Steigmann, R. W. Ogden, Proc. Roy. Soc. A, 455, 437 (1999)

11. V.A. Eremeyev, G. Rosi, S. Naili, Mech. Res. Commun., 74, 8 (2016)

12. V.A. Eremeyev, G. Rosi, S. Naili, Math. Mech. Solid., 1081286518769960 (2018)

13. Yu.Ya. Gotlib, A.V. Maximov, Polymer Sci., A, 34, 902 (1992) 
14. R. A. Gerasimov, V. A. Eremeyev, T. O. Petrova, V. I. Egorov, O. G. Maksimova, A. V. Maksimov, J. Phys.: Conf. Series, 738, 012100 (2016)

15. R. A. Gerasimov, O. G. Maksimova, T. O. Petrova, V. A. Eremeyev, A. V. Maksimov, Adv. Struct. Mater., 59, 55 (2017)

16. C. Boutin, F. dell'Isola, I. Giorgio, L Placidi, Math. Mech. Complex Syst., 5, 127 (2017)

17. L. Placidi, U. Andreaus, I. Giorgio, J. Eng. Math., 103, 1 (2017)

18. V. A. Eremeyev, F. dell'Isola, C. Boutin, D. Steigmann, J. Elast. 132, 175 (2018)

19. V.A. Eremeyev, Adv. Struct. Mater., 46, 153 (2017)

20. F. dell'Isola, I. Giorgio, M. Pawlikowski, N. Rizzi, Proc. Roy. Soc. A., 472, 20150790 (2016)

21. F. dell'Isola, P. Seppecher, J. J.Alibert, et al, Continuum Mech. Thermodyn., https://doi.org/10.1007/s00161-018-0689-8 (2018) 